MS16-P20

\section{Crystallization of carbonate minerals in organic matrices: Influence of hydrogel strength and magnesium doping on hydrogel-mineral organization in the mesocrystal composites}

Erika Griesshaber ${ }^{1}$, Lurdes Fernandez Diaz ${ }^{2}$, Fitriana Nindiyasari ${ }^{3}$, Xiaofei Yin ${ }^{1}$, Martina Greiner ${ }^{1}$, Andreas Ziegler ${ }^{4}$, Wolfgang W. Schmahl ${ }^{1}$

1. Department of Earth and Environment Sciences, LMU Munich, Munich, Germany

2. Instituto de Geociencias, Universidad Complutense Madrid, Madrid, Spain

3. NRG, Le Petten, Netherlands (Holland, Europe)

4. Center for Electron Microscopy University of Ulm, Ulm, Germany email: E.Griesshaber@Irz.uni-muenchen.de

Mineralized structures generated under biological control are functional tissues and are recognized as prototypes for man-made materials. Biological hard tissues are hierarchical composites and comprise two distinct components: compliant biopolymers that are reinforced by stiff carbonate and/or phosphate minerals.

In order to understand the composite nature and architecture of biologically mineralized hard tissues we conducted biomimetic crystal growth experiments in hydrogel matrices using the double diffusion experimental set-up. We characterized the obtained hydrogel-carbonate composites with FE-SEM, XRD, Rietveld analysis, micro-Raman spectroscopy, electron backscatter diffraction (EBSD). We investigated for two different hydrogels (gelatine, silica) the effect of gel solid content variation in Mg-bearing and Mg-absent growth environments on gel occlusion, gel distribution within the composite aggregate, hierarchy formation for the gel and the mineral and gel fabric and carbonate crystal organization $(1,2,3,4)$. Even though being artificial polymers, hydrogels mimic to some extent biogenic matrices, as they compartmentalize space, thus control, diffusion rates, local concentration of solutes and supersaturation. As each hydrogel has different chemical characteristics, they act differently in promoting or inhibiting crystallization.

We show that gelatin gel exerts most, while silica gel the least influence on calcite organization and aggregate formation (Figure 1). Irrespective of their solid content, all aggregates incorporate gel, which is inhomogenously distributed within the mineral. The mineral fraction in the aggregates is present in a mosaic and mesocrystal constitution. The presence of $\mathrm{Mg}^{2+}$ adds complexity to the internal structuring of the mineral. We show that two independent mechanisms are responsible for the impact of $\mathrm{Mg}^{2+}$ on composite organization. (1.) Addition of $\mathrm{Mg}^{2+}$ reduces the yield-strength of the gels by about $50 \%$. While gelatin gel shows continuous strain hardening, silica-gel weakens after reaching a shear strength maximum; the strain shifts by $350 \%$ to higher values. (2.) Incorporation of $\mathrm{Mg}^{2+}$ in the growing calcite increases its solubility, decreases crystallization pressure and introduces small angle grain boundaries that leads to split growth and ultimately to spherulitic growth. We show that $\mathrm{Mg}$ not only influences the organization of the mineral but also the structure of the gel matrix within the aggregates. With increasing $\mathrm{Mg}$ content the gel structure within the mineral changes from compact gel membranes to finely dispersed networks of gelatine fibres. This induces the large variety of calcite crystal co- and misorientation patterns in the investigated composites. We obtain a wide range of material design principles with some matching features of functional biological hard tissues.
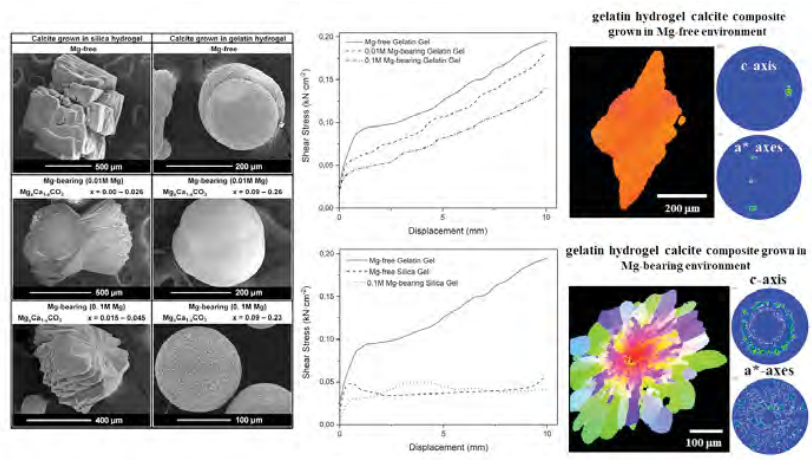

References:

(1) 1. F. Nindiyasari, L. Fernandez-Diaz, E. Griesshaber, J. M. Astilleros, N. Sanchez-Pastor, W. W. Schmahl 2014. Crystal Growth and Design, 14, 1531-1542.

(2) 2. F. Nindiyasari,E. Griesshaber, L. Fernandez-Diaz, J. M. Astilleros, N. Sanchez-Pastor, A. Ziegler, W. W. Schmahl 2014. Crystal Growth and Design, 14, 4790-4802.

(3) 3. F. Nindiyasari, A. Ziegler, E. Griesshaber, L. Fernandez-Diaz, P. Walther, W. W. Schmahl 2015. Crystal Growth and Design, 2667-2685.

(4) 4. X. Yin, E. Griesshaber, L. Fernendez-Diaz, A. Zieger, F. J. Garcia-Garcia, W. W. Schmahl 2018. Formation of hydrogel-calcite composites in mixed gelatin/agarose hydrogels: Patterns of mineral assembly aggregate formation principles. Manuscript to be submitted to Crystal Growth and Design.

Keywords: Biomimetic composites, material properties, mesocrystals 